\title{
Recent Issues on Body Composition Imaging for Sarcopenia Evaluation
}

\author{
Koeun Lee, $M D^{1 *}$, Yongbin Shin, $\mathrm{MS}^{1 *}$, Jimi Huh, MD, PhD ${ }^{2}$, Yu Sub Sung, PhD ${ }^{1}$, In-Seob Lee, MD, PhD ${ }^{3}$, \\ Kwon-Ha Yoon, MD, PhD ${ }^{4}$, Kyung Won Kim, MD, $\mathrm{PhD}^{1}$
}

${ }^{1}$ Department of Radiology, Asan Image Metrics, Asan Medical Center, University of Ulsan College of Medicine, Seoul, Korea; ${ }^{2}$ Department of Radiology, Ajou University School of Medicine \& Graduate School of Medicine, Ajou University Medical Center, Suwon, Korea; ${ }^{3}$ Department of Surgery, Asan Medical Center, University of Ulsan College of Medicine, Seoul, Korea; ${ }^{4}$ Department of Radiology, Wonkwang University College of Medicine, Wonkwang University Hospital, Iksan, Korea

Recently, sarcopenia has garnered renewed interest. Sarcopenia is a disease characterized by decreased skeletal muscle mass and strength/function, which can impair the quality of life and increase physical disability, adverse metabolic effects, and mortality. Imaging tools for evaluating and diagnosing sarcopenia have developed rapidly. Radiologists should be aware of sarcopenia and its clinical implications. We review current knowledge about sarcopenia, its pathophysiological impact, and advantages and disadvantages of methods for evaluation of sarcopenia focusing on body composition imaging modalities such as whole-body dual-energy X-ray absorptiometry, CT, and MRI. Controversial issues are discussed, including the lack of consensus and standardization of the disease definition, imaging modality, measurement methods, and diagnostic cutoff points. Keywords: Body composition; Computed tomography; Magnetic resonance imaging; Dual-energy X-ray absorptiometry; Sarcopenia

\section{INTRODUCTION}

Assessment of body composition typically refers to the quantification of body fat and muscle mass, and it is most commonly assessed by medical imaging. In the past few decades, body composition imaging has mostly focused on evaluation of body fat, such as visceral fat or subcutaneous fat, because obesity and its clinical

Received July 23, 2018; accepted after revision October 15, 2018. This study was supported by a grant of the Korea Health Technology R\&D Project through the Korea Health Industry Development Institute (KHIDI), funded by the Ministry of Health \& Welfare, Republic of Korea (grant number : HI18C1216). *These authors contributed equally to this work. Corresponding author: Jimi Huh, MD, PhD, Department of Radiology, Ajou University School of Medicine \& Graduate School of Medicine, Ajou University Medical Center, 164 Worldcup-ro, Yeongtong-gu, Suwon 16499, Korea.

- Tel: (8231) 219-4369 - Fax: (8231) 219-5862

- E-mail: jimihuh.rad@gmail.com

This is an Open Access article distributed under the terms of the Creative Commons Attribution Non-Commercial License (https://creativecommons.org/licenses/by-nc/4.0) which permits unrestricted non-commercial use, distribution, and reproduction in any medium, provided the original work is properly cited. implications has been extensively investigated $(1,2)$. However, in the past decade, the importance of muscle mass has been emphasized and has become a focal point for clinical research (3). Indeed, in 2016, sarcopenia itself was classified by the International Classification of Diseases (ICD-10-CM), with the code M62.84.

Sarcopenia, a term first introduced in 1984 by Rosenberg, refers to age-related loss of muscle mass, and is thus a type of geriatric syndrome (4). Many studies have proven its relationship with physical impairment and poorer quality of life, and increased morbidity, mortality, and health care costs. Recently, the concept of sarcopenia has been extended to various diseases, beyond merely being considered as a geriatric syndrome (5). In particular, the influence of sarcopenia on morbidity/mortality in cancer patients treated with chemotherapy or major surgery has been extensively investigated.

Currently, there are diverse methods for evaluating muscle mass by imaging, and these methods must be standardized. Such standardization requires a massive accumulation of evidence and efforts to achieve a worldwide consensus. Evidence and consensus are only beginning to mount 
at present, indicating that the imaging modalities and methods used to diagnose sarcopenia will remain varied for a while to come. The role of imaging techniques has rapidly increased in the field of sarcopenia. Accordingly, radiologists should be aware of sarcopenia and its clinical implications.

In this paper, we aim to present a comprehensive review of the role of the different imaging modalities currently available for the non-invasive assessment of skeletal muscle. We also discuss several controversial issues in sarcopenia and their clinical relevance.

\section{Terminology and Definition}

Sarcopenia, derived from the Greek words for flesh (sarx) and loss (penia), is a condition of decreased skeletal muscle mass that can lead to a decline in physical ability (4). There is a lack of worldwide agreement on the definition of sarcopenia. There are three major consensus groups (4). Currently, the most commonly accepted and used definition is from the European Working Group on sarcopenia in older people (EWGSOP, the Sarcopenia Working Group), created in 2010. The EWGSOP recommends using the presence of both low muscle mass and low muscle function (strength or performance) for the diagnosis of sarcopenia (4). Regarding the severity of sarcopenia, EWGSOP has suggested a staging concept that can be helpful in clinical management: "presarcopenia," "sarcopenia," and "severe sarcopenia" (5).

Sarcopenia is often confused with the terms frailty and cachexia. These conditions show marked overlap because sarcopenia (i.e., decreased muscle mass and muscle function) is a major component of frailty and cachexia. Frailty is another geriatric syndrome characterized by reduced homeostatic reserves (resilience) that carries an increased risk of poor health outcomes, including falls, incident disability, hospitalization, and mortality (6). Fried et al. have defined frailty as meeting three of five of the following criteria: low grip strength, low energy, slowed walking speed, low physical activity, and unintentional weight loss (7). Frailty is not exclusively related to muscle itself, but is more complex and is often preceded by sarcopenia $(7,8)$. Cachexia is a progressive and involuntary loss of skeletal muscle mass and fat mass due to severe disease, such as cancer, chronic obstructive pulmonary disease, chronic heart failure, and chronic kidney disease. Cachexia is a form of secondary sarcopenia caused by severe disease, but it is characterized by both weight loss and malnutrition, due to systemic metabolic imbalances and inflammation, with prominent overexpression of proinflammatory cytokines, which can cause proteolysis and lipolysis (9).

\section{Pathophysiology and Clinical Impact}

Sarcopenia has a high prevalence $(29-33 \%)$ in community-dwelling populations and long-term care populations (10). In general, aging leads to changes in muscle fibers and $\alpha$-motor neurons. Decreased production of growth hormone and sex-steroid hormones with aging also can aggravate muscle changes. Sarcopenia can be primary (resulting from aging) or secondary (resulting from early life developmental influences, malnutrition, bed rest or little physical activity, and certain treatments), although in most cases, as sarcopenia is a multi-faceted syndrome, it may not be possible to categorize it as primary or secondary (11).

The concept of muscle function is being revolutionized. In the past, skeletal muscle was considered an organ related to mobility, with a mechanical function. It is now known that muscle has various functions, such as metabolic, endocrine, neurological, and even psychological functions (Fig. 1). The concept of "muscle as a secretory organ" has been proposed, as studies have shown the multi-systemic effects of muscle via control of myokine release (12).

Myokines are cytokines and other peptides, such as IL- 6 and brain-derived neurotrophic factor (BDNF), which perform actions by communicating with other organs (12). For example, IL- 6 promotes glucose production in the liver, lipolysis in adipose tissue, increased pancreatic beta-cell viability, and insulin secretion, suggesting that it has an important role in maintaining glucose homeostasis. BDNF plays a crucial role in regulating the growth and maintenance of neurons; therefore, it influences learning, memory, and even mood (13).

Many studies have reported that myokine production is related to muscle contraction. Accordingly, physical inactivity may cause imbalances between these substances, which leads to a pro-inflammatory status and metabolic dysfunction that promotes the critical cascade of sarcopenia and fat accumulation (14). In connection with this new pathophysiological concept, the impact of sarcopenia on chronic disease is also being investigated. Skeletal muscle is also strongly related to energy metabolism, since it is the largest organ that consumes glucose as an energy source. Thus, sarcopenia is related to metabolic syndromes and various cardiovascular diseases (15-17). In addition to the 
metabolic effects, sarcopenia can lead to physical disability, falling, fracture, hospitalization, depression, poor quality of life, increased health care costs, and notably increased mortality $(1,2)$. Currently, the evidence of the impact of sarcopenia in various disease settings and on health status is rapidly accumulating (Fig. 2). First, the impact of sarcopenia on the outcomes of various treatments, such as major surgery and cancer chemotherapy, has been extensively investigated. Systematic reviews of patients operated for gastrointestinal and hepato-pancreato-biliary

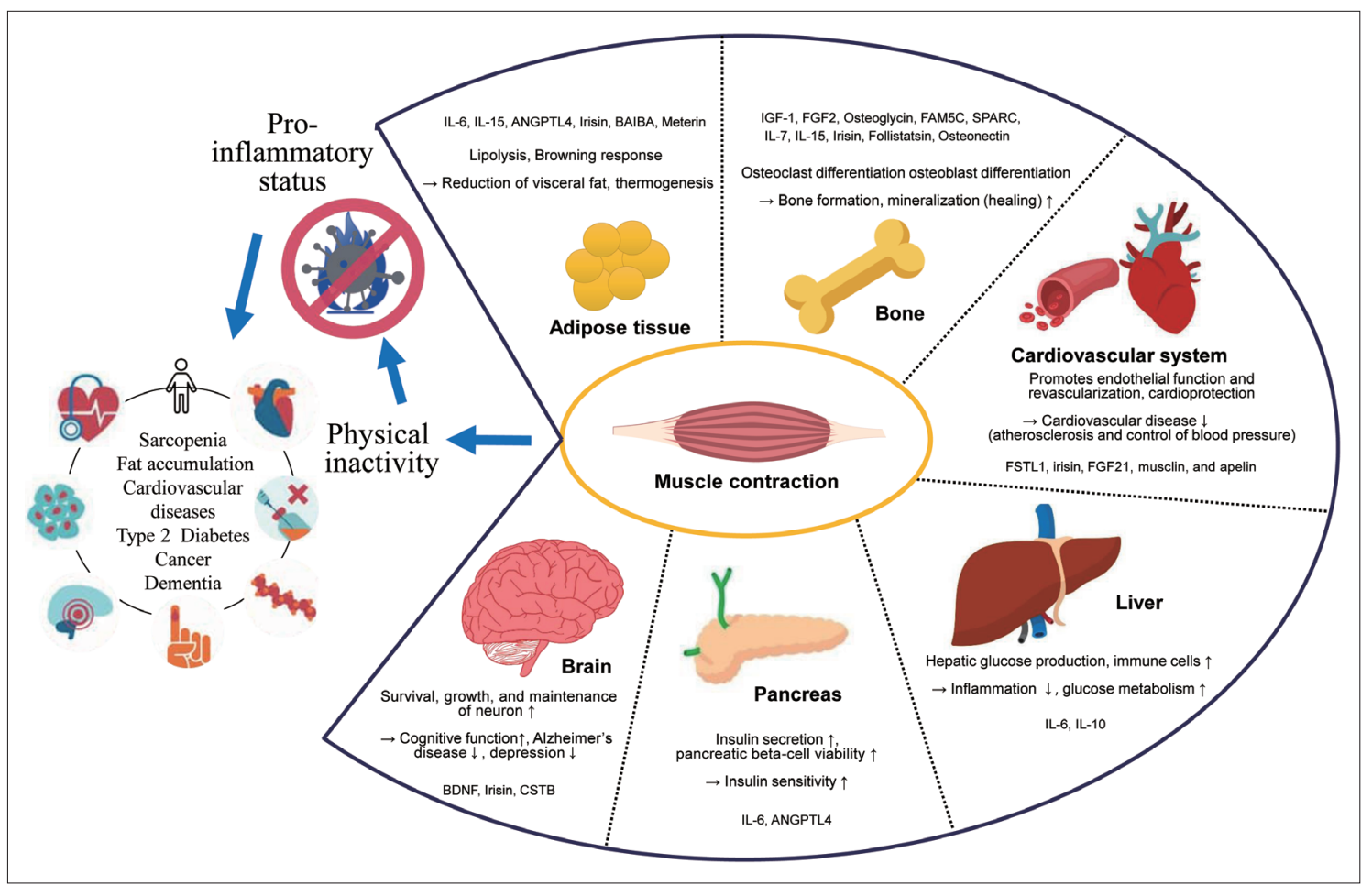

Fig. 1. Muscle as secretory organ. Various myokines released through muscle contraction affecting anatomically distant organs, which could explain association between sedentary behavior and many chronic diseases. ANGPTL4 = angiopoietin-like 4 , BAIBA = $\beta$-aminoisobutyric acid, $\mathrm{BDNF}=$ brain-derived neurotrophic factor, CSTB $=$ cathepsin B, FAM5C = family with sequence similarity 5 , FGF2 = fibroblast growth factor 2 , FGF21 = fibroblast growth factor 21, FSTL1 = follistatin-related protein 1, IGF1 = insulin-like growth factor 1, SPARC = secreted protein acidic and rich in cysteine, IL-6 = interleukin-6, IL-7 = interleukin-7, IL-10 = interleukin-10, IL-15 = interleukin-15

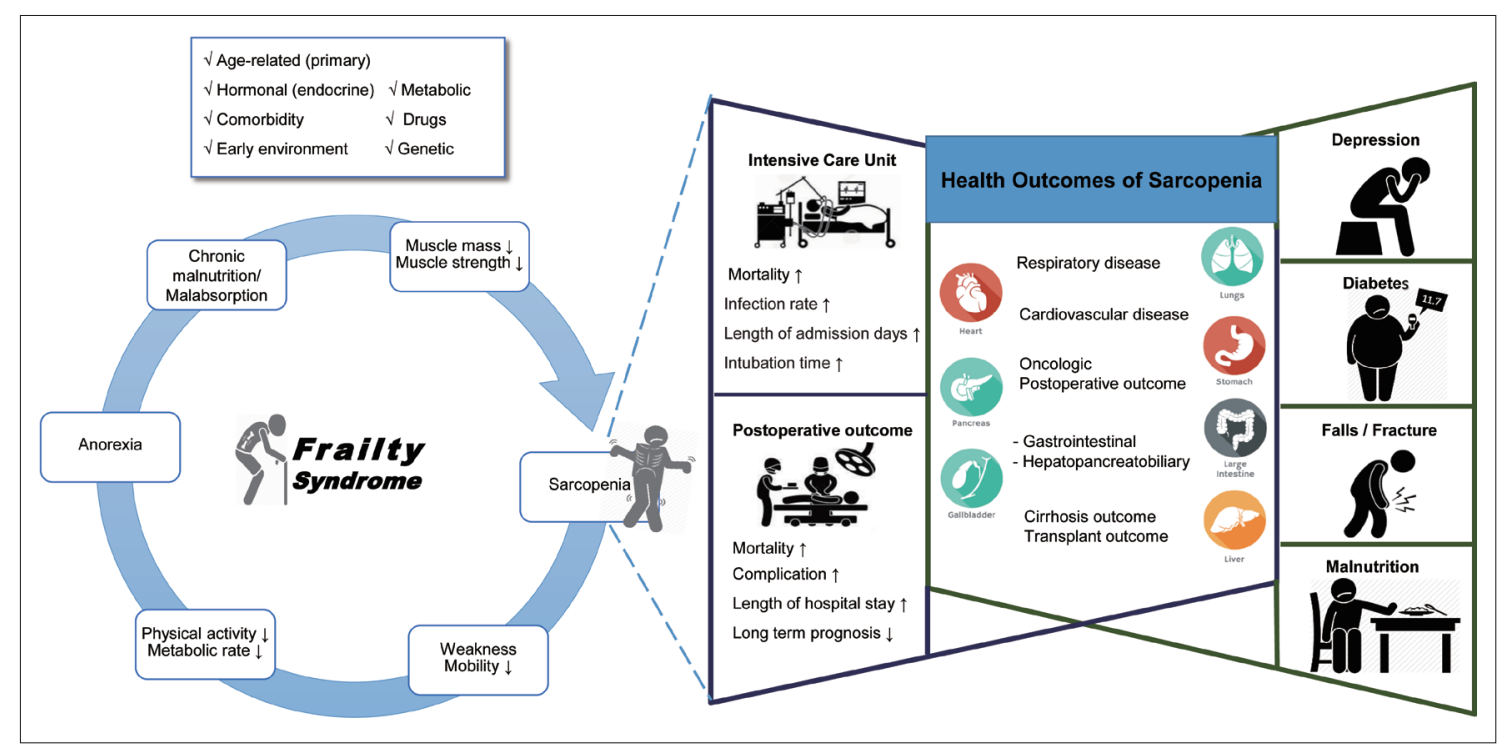

Fig. 2. Sarcopenia cascade. Various cause of sarcopenia entering vicious cycle, resulting in physical disability, falling, fracture, hospitalization, depression, poor quality of life, increased health care costs, adverse metabolic effects, and notably increased mortality. 
malignancies have reported impaired overall survival and increased postoperative morbidity in patients with sarcopenia $(18,19)$. Sarcopenia is also associated with increased mortality in patients who were treated with liver transplantation (20), selective and non-abdominal aortic aneurysm repair $(21,22)$, and pancreatic adenocarcinoma treatment (23). In addition, in the setting of an intensive care unit and emergency care, sarcopenia has been reported to be a significant predictor of mortality $(3,24,25)$.

\section{Evaluation of Muscle Quality and Myosteatosis}

One of the critical issues in sarcopenia is the discrepancy between muscle mass and muscle function, which might be mainly attributed to the fatty degeneration or fatty infiltration of the muscle; i.e., myosteatosis. Myosteatosis is regarded as a part of the aging process or muscle disuse that is independent of obesity (26). Myosteatosis has been proven to be associated with decreased muscle strength and mobility, and increased disability (27-29).

Recent studies have found evidence indicating that disuse leads to fat infiltration, which influences muscle fiber transition from type II to type I, thus resulting in impaired contractile capacity with decreased muscle power (30). During this process, metabolic pathways, such as leptin signaling, inflammatory adipokine, sex steroid, and glucocorticoid signaling, and glucose metabolic pathways, are involved. Thus, myosteatosis is not merely a problem of muscle, but is closely related to metabolic and systemic dysfunction (26). Several studies have reported that accumulation of muscle fat decreases insulin sensitivity, leading to type 2 diabetes, impairing the capacity for normal protein synthesis in skeletal muscle, leading to loss of muscle mass and strength, which results in increased disability and mortality among older adults (31-33).

\section{Overview of Assessment Techniques and Current Issues}

There are many methods to evaluate muscle mass, including anthropometry, bioelectrical impedance analysis (BIA), and medical imaging (Table 1). Anthropometry assessments, such as body-mass index (BMI), skin-fold thickness, and body circumference (e.g., waist, thigh, and calf), are simple and readily available in any clinical setting, but may not be sufficiently accurate to evaluate muscle mass. BIA, which calculates the muscle mass and fat mass based on different conduction of current through tissues, is low cost and simple to use. However, measurement errors may be present depending on differences in hydration level, exercise status, or even food intake (3). In a study aiming to validate the accuracy of BIA in body composition prediction, dual-energy X-ray absorptiometry (DXA) and MRI showed that BIA devices tend to overestimate at lower levels and underestimate at higher levels, while being relatively accurate at predicting fat mass and skeletal muscle mass percentages.

Medical imaging is considered an accurate and reliable method for quantifying muscle and fat mass and it discerns the spatial distribution of fat and muscle, enabling diagnosis and grading of sarcopenia, obesity, or both (i.e., sarcopenic obesity). Medical imaging is useful for tracking longitudinal changes in muscle and fat; thus, it can be used in clinical trials as a treatment assessment tool (2, $3)$. The common imaging modalities for evaluating muscle mass include whole-body DXA, CT, and MRI. Use of the ultrasonography (US) is limited in some clinical settings (3).

Currently, there are many issues regarding the utilization of medical imaging for body composition assessment. There is no consensus on which imaging modality to choose, which body region to measure, which guideline for image acquisition to follow, or what cutoff points should be used to evaluate skeletal muscle quality and quantity. Standardization of the diagnostic index and its diagnostic cutoffs, based on sufficient evidence and international consensus, remains necessary. One important consideration is ethnicity. The cutoff points appear to differ between Asian and Caucasian populations, due to differences in ethnicity, adiposity, size, and lifestyle, which may also affect the prevalence of sarcopenia.

\section{Whole-Body DXA}

\section{Techniques}

DXA measures the absorption of two X-ray photon energies, typically near 40-47 keV and 70-80 keV. The measurement of transmitted intensities at two photon energies enables the differentiation of bone, fat mass, and soft tissue lean mass (non-bone and non-fat soft tissue), based on different $X$-ray attenuation of tissues (2). DXA utilizes two-dimensional projection, i.e., it provides a snapshot of the body composition in the whole body. In addition, DXA shows the amount of fat and lean tissue in each body part, such as the left arm or right leg. 
Table 1. Body Composition Imaging

\begin{tabular}{|c|c|c|c|c|}
\hline Modality & Commonly Used Parameters & Pros & Cons & $\begin{array}{c}\text { Error of Lean Mass } \\
\text { Estimate }\end{array}$ \\
\hline DXA & $\begin{array}{l}\text { Whole-body lean mass } \\
\text { Appendicular lean mass } \\
\text { Apendicular lean mass/height } \\
\text { squared }\end{array}$ & $\begin{array}{l}\text { Inexpensive } \\
\text { Low radiation exposure } \\
\text { Short image acquisition time } \\
\text { Simultaneous measurement of } \\
\text { whole-body fat mass and bone } \\
\text { mass }\end{array}$ & $\begin{array}{l}\text { Lack of portability } \\
\text { 2-dimensional data } \\
\text { No differentiation between } \\
\text { Subcutaneous and visceral fat } \\
\text { Does not include trunk muscles }\end{array}$ & $<5 \%$ \\
\hline CT & $\begin{array}{l}\text { Muscle size (CSA, volume) } \\
\text { Muscle echo intensity }\end{array}$ & $\begin{array}{l}\text { High accuracy and reproducible } \\
\text { results } \\
\text { Simultaneous measurement of } \\
\text { lean body mass, visceral and } \\
\text { subcutaneous fat } \\
\text { Differentiate between fat and } \\
\text { fat-free mass }\end{array}$ & $\begin{array}{l}\text { Expensive } \\
\text { High complexity } \\
\text { Radiation exposure }\end{array}$ & $<8.5 \%$ \\
\hline MRI & $\begin{array}{l}\text { Muscle edema, atrophy, fatty } \\
\text { infiltration } \\
\text { Muscle size (CSA, volume) } \\
\text { Muscle adipose tissue content }\end{array}$ & $\begin{array}{l}\text { No radiation exposure } \\
\text { Best spatial resolution } \\
\text { Body mass composition } \\
\text { differentiation } \\
\text { Suitable for long-term follow-up, } \\
\text { progression monitoring } \\
\text { Capable of detecting changes in } \\
\text { muscle structure }\end{array}$ & $\begin{array}{l}\text { Expensive } \\
\text { High complexity } \\
\text { Limited access } \\
\text { Longer image acquisition time } \\
\text { Some patients with } \\
\text { contraindications } \\
\text { Lack of standardized assessment } \\
\text { protocol }\end{array}$ & $6-8.5 \%$ \\
\hline US & $\begin{array}{l}\text { Muscle size (CSA, volume) } \\
\text { Muscle thickness } \\
\text { Muscle attenuation } \\
\text { Echo intensity } \\
\text { Fascicle length } \\
\text { Pennation angle }\end{array}$ & $\begin{array}{l}\text { Inexpensive } \\
\text { No radiation exposure } \\
\text { Short image acquisition time } \\
\text { Portable } \\
\text { Real time visualization of target } \\
\text { structure }\end{array}$ & $\begin{array}{l}\text { Operator skills and training } \\
\text { required } \\
\text { Reliability and accuracy depend } \\
\text { on operator } \\
\text { Poor reproducibility and accuracy }\end{array}$ & \\
\hline
\end{tabular}

$\mathrm{CSA}=$ cross-sectional area, DXA = dual-energy X-ray absorptiometry, US = ultrasonography

DXA has the advantage of a relatively low radiation exposure, as low as $0.001 \mathrm{mSv}$, which is much less than that with standard chest radiography. In addition, DXA has low costs as compared to those of a CT scan, and is not limited by its operational complexity (34). Limitations of DXA include a lack of accuracy in estimating truncal fat and muscle, due to the presence of intra-abdominal solid organs and the bowel. In particular, visceral fat measurement is greatly hampered, frequently requiring additional crosssectional imaging, such as CT or MRI. In body composition studies, measuring intra-abdominal fat volume is especially important because visceral fat is metabolically more active than subcutaneous fat $(35,36)$. In addition, trunk muscles, such as chest and back muscles, are some of the most prominent muscles in the body but are very difficult to evaluate on DXA. Therefore, measurements of fat mass and muscle mass are generally derived from arms and legs, which might over/underestimate the extent of sarcopenia and obesity. In addition, muscle and fat measurement can be biased by hydration status and the presence of edema (35). Nevertheless, the ability to evaluate the whole body (trunk and extremities) very easily is the most attractive characteristic of DXA as compared to CT and MRI (2). This is well documented, as DXA is the preferred body composition imaging modality in many guidelines for diagnosis of sarcopenia, including the EWGSOP (37).

\section{Diagnostic Index and Criteria}

With the increasing interest and use of DXA in the field of sarcopenia, many different indices have been developed and utilized in guidelines, as summarized in Table 2. For measurement of skeletal muscle mass, appendicular skeletal muscle mass (ASM), which is the sum of the lean muscle mass of both arms and legs, is generally used as a basic index, as illustrated in Figure 3. Since muscle mass is highly correlated with total body size (weight and height in 
general), instead of using the absolute ASM directly, ASM is adjusted in few different ways, namely by using the height squared (ASM/ht ${ }^{2}$ ), weight (ASM/wt), or BMI (ASM/BMI). Among these, the most commonly used index is ASM/ht', which was adopted in the EWGSOP guidelines and referred to as Skeletal Muscle Index (15).

The ASM $/ \mathrm{ht}^{2}$ was first suggested by Baumgartner et al. (38) in the New Mexico Elder Health Survey. In this study, the ASM $/ \mathrm{ht}^{2}$ index was proven to have a significant correlation with clinical outcomes, such as frailty. Thereafter, several international guidelines have adopted the ASM $/ \mathrm{ht}^{2}$ as a diagnostic index for sarcopenia (Table 2) (15). The first study to set an example for cutoff points was that by Baumgartner et al. (38), who used ASM two standard deviations below the mean of the ASM index measured by DXA in a young reference group: $7.26 \mathrm{~kg} / \mathrm{m}^{2}$ for men and $5.45 \mathrm{~kg} / \mathrm{m}^{2}$ for women (5). Since then, many groups have followed the example of Baumgartner et al. (38) and have proposed cutoff points within their reference group, as shown in Table $2(39,40)$.

The limitation of this ASM $/ \mathrm{ht}^{2}$ index is that subjects with a high BMI might be masked as not sarcopenic, due to a larger amount of fat mass. Recently, in 2014, the Foundation for the National Institutes of Health Sarcopenia Project proposed a BMI-adjusted index, ASM/BMI, based on large and diverse population-based studies. In their study, they performed a statistical analysis to show which muscle mass index best correlates with weakness and slowness (41). The ASM/BMI has been increasingly used but has not gained full acceptance yet (15).

\section{CT and MRI}

\section{CT Techniques}

With great advances in its technology, CT has become the most widely used cross-sectional imaging modality, and is readily available worldwide. In particular, CT has become the standard diagnostic tool in many clinical settings for procedures such as cancer treatment, major surgery, and assessment of vascular disease. Thus, the body composition analysis in clinically acquired CT scans are useful in patients who require $\mathrm{CT}$ for disease management (42).

CT can accurately differentiate between fat and muscle tissue using the specific attenuation of each kind of tissue and it provides very detailed anatomical information (Fig. 4). For example, fat tissue typically ranges from -30 to -190 Hounsfield units. Due to the measurement accuracy of fat and muscle, CT has been considered the gold standard for investigating quantitative and qualitative changes in muscle and fat, especially for the trunk area where DXA is

Table 2. Muscle Mass Criteria by Various Study Groups

\begin{tabular}{|c|c|c|c|c|c|}
\hline \multirow{2}{*}{ Study Group } & \multicolumn{2}{|c|}{ Muscle Mass } & \multicolumn{2}{|c|}{ Muscle Strength } & \multirow{2}{*}{ Physical Performance } \\
\hline & Male & Female & Male & Female & \\
\hline EWGSOP 2010 & $\begin{array}{l}\text { DXA } \\
\text { ASM } / \mathrm{ht}^{2} \leq 7.23 \mathrm{~kg} / \mathrm{m}^{2}\end{array}$ & $\begin{array}{l}\text { DXA } \\
\text { ASM } / \mathrm{ht}^{2} \leq 5.67 \mathrm{~kg} / \mathrm{m}^{2}\end{array}$ & $\begin{array}{l}\text { Grip strength } \\
<30 \mathrm{~kg}\end{array}$ & $\begin{array}{l}\text { Grip strength } \\
<20 \mathrm{~kg}\end{array}$ & Gait speed $\leq 0.8 \mathrm{~m} / \mathrm{s}$ \\
\hline IWGS 2011 & $\begin{array}{l}\text { DXA } \\
\text { ASM } / \mathrm{ht}^{2} \leq 7.23 \mathrm{~kg} / \mathrm{m}^{2}\end{array}$ & $\begin{array}{l}\text { DXA } \\
\text { ASM } / \mathrm{ht}^{2} \leq 5.67 \mathrm{~kg} / \mathrm{m}^{2}\end{array}$ & & & Gait speed $\leq 1.0 \mathrm{~m} / \mathrm{s}$ \\
\hline AWGS 2014 & $\begin{array}{l}\text { DXA } \\
\text { ASM } / \mathrm{ht}^{2} \leq 7.0 \mathrm{~kg} / \mathrm{m}^{2}\end{array}$ & $\begin{array}{l}\text { DXA } \\
\text { ASM } / \mathrm{ht}^{2} \leq 5.4 \mathrm{~kg} / \mathrm{m}^{2}\end{array}$ & $\begin{array}{l}\text { Grip strength } \\
<26 \mathrm{~kg}\end{array}$ & $\begin{array}{l}\text { Grip strength } \\
<18 \mathrm{~kg}\end{array}$ & Gait speed $\leq 0.8 \mathrm{~m} / \mathrm{s}$ \\
\hline FNIHSP 2014 & $\begin{array}{l}\text { DXA } \\
\text { ASM/BMI } \leq 0.789\end{array}$ & $\begin{array}{l}\text { DXA } \\
\text { ASM/BMI } \leq 0.512\end{array}$ & $\begin{array}{l}\text { Grip strength } \\
<26 \mathrm{~kg}\end{array}$ & $\begin{array}{l}\text { Grip strength } \\
<16 \mathrm{~kg}\end{array}$ & Gait speed $\leq 0.8 \mathrm{~m} / \mathrm{s}$ \\
\hline $\begin{array}{l}\text { ESPEN special } \\
\text { interest group }\end{array}$ & $\begin{array}{l}\text { Muscle mass } \% \geq 2 \text { SD } \\
\text { individuals aged } 18-3 \\
\text { cohort }\end{array}$ & $\begin{array}{l}\text { pelow mean in } \\
9 \text { years in NHANES III }\end{array}$ & & & $\begin{array}{l}\text { Walking speed } \leq 0.8 \mathrm{~m} / \mathrm{s} \text { in } 4 \text { min } \\
\text { test or reduced performance } \\
\text { in any functional test used } \\
\text { for comprehensive geriatric } \\
\text { assessment }\end{array}$ \\
\hline $\begin{array}{l}\text { Society of sarcopenia, } \\
\text { cahexia and wasting } \\
\text { disorders }\end{array}$ & $\begin{array}{l}\text { ASM } / \text { ht }^{2} \geq 2 \text { SD below } n \\
\text { aged between } 20 \text { and } \\
\text { group }\end{array}$ & $\begin{array}{l}\text { nean of healthy persons } \\
30 \text { years of same ethnic }\end{array}$ & & & $\begin{array}{l}\text { Gait speed } \leq 1.0 \mathrm{~m} / \mathrm{s} \text { or } \\
\text { walking distance }<400 \mathrm{~m} \\
\text { during } 6 \text { min walk }\end{array}$ \\
\hline
\end{tabular}

ASM = appendicular skeletal muscle, AWGS = Asian Working Group for sarcopenia, BMI = body-mass index, ESPEN = European Society for Clinical Nutrition and Metabolism, EWGSOP = European Working Group on sarcopenia in older people, FNIHSP = Foundation for National Institutes of Health Sarcopenia Project, IWGS = International Working Group on sarcopenia, NHANES III = Third National Health and Nutrition Examination Survey, SD= standard deviation 
limited (37). In addition, the reliability of CT to evaluate quantitative and qualitative changes in fat and muscle mass has been well documented over the last 25 years $(2,43)$.

Beyond the mere quantification of the muscle mass, CT can evaluate the quality of muscle based on identifying the fat portion within the muscle. For example, decreased attenuation indicates an increased fat portion within the muscle; gross fat infiltration can be separated from the muscle fibers. This aspect of CT also makes it suitable for assessment of fat infiltration in muscle, known as
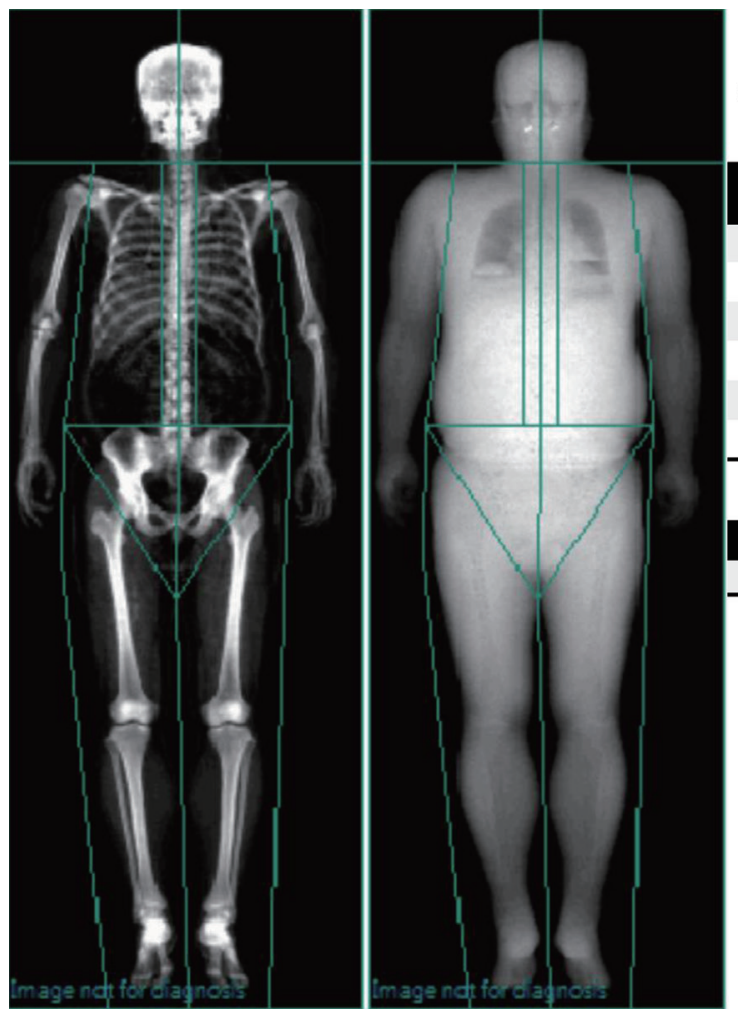

Height/Weight : $175.8 \mathrm{~cm} 95.0 \mathrm{~kg}$ Sex/Ethnic : Male Asian

BODY COMPOSITION

\begin{tabular}{cccccccc} 
Region & $\begin{array}{c}\text { Tissue } \\
(\% \text { Fat) }\end{array}$ & $\begin{array}{c}\text { Region } \\
(\% \text { Fat) }\end{array}$ & $\begin{array}{c}\text { Tissue } \\
(\mathbf{g})\end{array}$ & $\begin{array}{c}\text { Fat } \\
(\mathbf{g})\end{array}$ & $\begin{array}{c}\text { Lean } \\
(\mathbf{g})\end{array}$ & $\begin{array}{c}\text { BMC } \\
(\mathbf{g})\end{array}$ & $\begin{array}{c}\text { Total Mass } \\
(\mathbf{k g})\end{array}$ \\
\hline Arms & 35.0 & 33.6 & 8563 & 3001 & 5562 & 365 & 8.9 \\
\hline Legs & 32.1 & 30.8 & 27052 & 8671 & 18381 & 1125 & 28.2 \\
Trunk & 50.5 & 49.3 & 50363 & 25415 & 24948 & 1206 & 51.6 \\
Android & 57.0 & 56.5 & 8686 & 4950 & 3736 & 68 & 8.8 \\
Gynoid & 39.6 & 38.7 & 13823 & 5478 & 8345 & 325 & 14.1 \\
Total & 42.0 & 40.6 & 91280 & 38333 & 52947 & 3228 & 94.5 \\
\hline
\end{tabular}

FAT MASS RATIOS

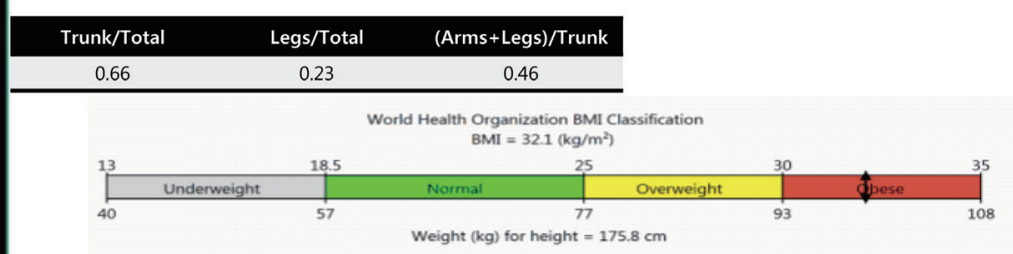

$$
\begin{aligned}
& \text { ASM }=23.943 \mathrm{Kg} \\
& \text { ASM } / \text { height }^{2}=7.818 \mathrm{Kg} / \mathrm{m}^{2} \\
& \text { ASM/weight }=0.252 \\
& \text { ASM/BMI }=0.771
\end{aligned}
$$

Fig. 3. Dual-energy x-ray absorptiometry results for body composition in healthy 47-year-old male. ASM is sum of lean muscle mass of both arms and legs. ASM is adjusted using height squared $\left(\right.$ ASM $\left./ h^{2}{ }^{2}\right)$, weight (ASM/wt), or BMI (ASM/BMI). ASM = appendicular skeletal muscle, $\mathrm{BMC}=$ bone mineral content, $\mathrm{BMI}=$ body-mass index

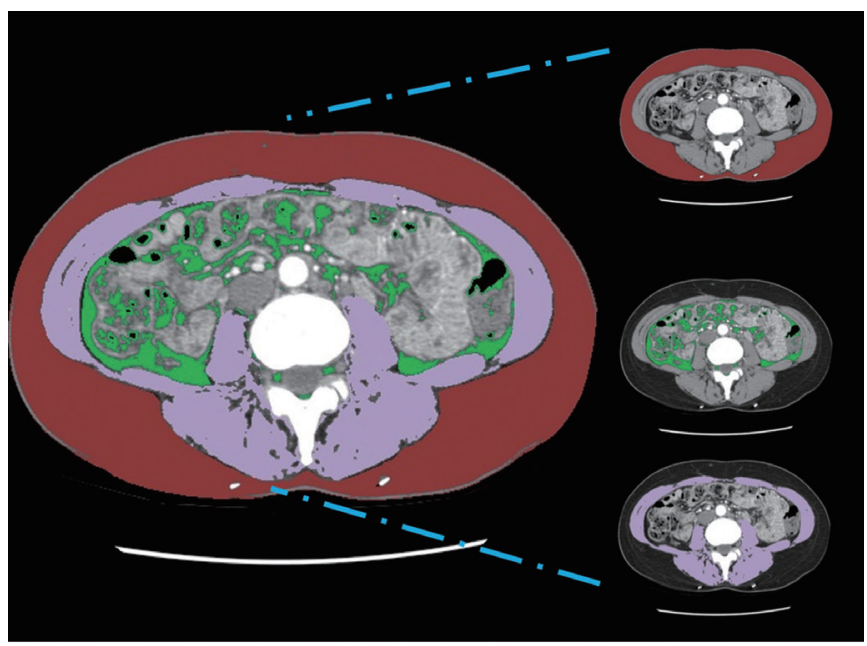

A

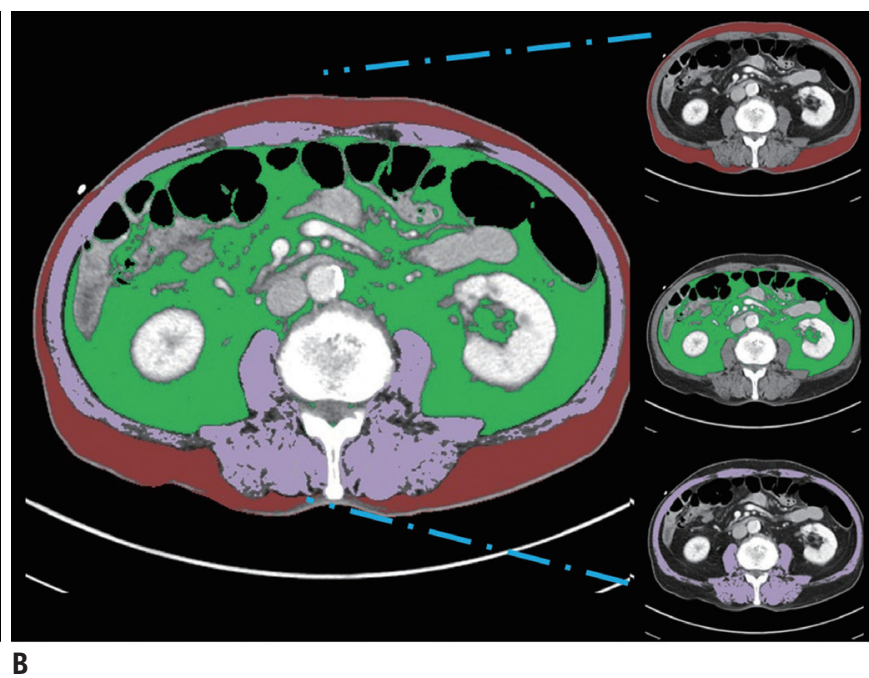

Subcutaneous fat

Fig. 4. Body composition evaluation CT image with artificial intelligence segmentation technique.

In clinically acquired axial CT images at L3 vertebral body level TAMA, visceral fat area, subcutaneous fat area are segmented in 43-year-old male (A), and 55-year-old obese male (B). TAMA = total abdominal muscle area 
myosteatosis (26). However, CT is limited in that it cannot directly measure the lipid content or distinguish between intra-myocellular fat and intermuscular fat.

The use of CT is limited by its high-dose radiation exposure and high cost. The effective radiation dose from a standard abdomen/pelvis CT is typically around $8 \mathrm{mSv}$, which is higher than the radiation exposure from the natural environment (2.5 mSv). Recently, many efforts have attempted to reduce radiation dose, such as by using a lowdose protocol or single-slice scanning. Indeed, Yoon et al.'s study (44) demonstrated that low-dose CT provides accurate and reproducible abdominal fat measurement, based on a phantom and human study. In addition, scanning with only a single slice can be performed for the evaluation of body composition; this markedly reduces the radiation dose, to less than $1 \mathrm{mSv}$ (42). Despite these efforts, radiation exposure is still high, which limits the use of CT for body composition assessment only. Therefore, in most studies using $\mathrm{CT}$ for body composition assessment, the CT images were clinically acquired during disease treatment or during the follow-up period (3).

\section{MRI Techniques}

MRI uses differences in the radiofrequency pulse sequence to distinguish between adipose tissue and fat-free mass. For example, a short $\mathrm{T} 1$ and a long $\mathrm{T} 2$ proton relaxation time has been shown to indicate adipose tissue (45). Like CT, MRI is also a cross-sectional imaging modality, enabling accurate measurement of body fat and muscle mass. Unlike CT, MRI has the advantage of no radiation exposure, making it more appropriate for long-term follow-up. In addition, MRI can assess detailed tissue structure and composition, facilitating quantification of muscle volume and quality from individual muscle groups. Notably, MRI can also provide information on edema, inflammation in muscle, fatty infiltration, fibrosis, and atrophy $(46,47)$. However, MRI is limited by its high cost and limited accessibility or availability. Its limitation also includes the long image acquisition time and operational complexity. Therefore, body composition assessment on MRI is performed when there are clinically acquired MRI images obtained during disease treatment or follow-up (47).

Regarding the evaluation of muscle quality and myosteatosis, MRI demonstrates the best contrast between adipose and muscle tissue (42) and recently was shown to have higher sensitivity for detecting early fatty replacement in muscles, with better visibility of anatomical details than
CT (48).

Magnetic resonance spectroscopy, a special MRI technique used to evaluate the chemical and molecular composition of tissue, can distinguish between intra-myocellular and extramyocellular fat (26). However, these imaging techniques are not fully validated yet, warranting further studies and evidence accumulation.

\section{Diagnostic Index and Criteria}

Like DXA, there is no consensus about the criteria or cutoff values, or even which indices should be used, for assessing skeletal muscle mass when using $\mathrm{CT} / \mathrm{MRI}$ for measuring sarcopenia. Many studies have proposed a diverse range of cutoff points for using CT/MRI images to evaluate sarcopenia. For example, for the total abdominal muscle area (TAMA) measurement at the $L 3$ vertebral level, there are two commonly used cutoff points. First, in Prado et al.'s study (49), sex-specific cutoffs were proposed: $52.4 \mathrm{~cm}^{2} / \mathrm{m}^{2}$ for men and $38.5 \mathrm{~cm}^{2} / \mathrm{m}^{2}$ for women, with patients below these values classified as sarcopenic. Second, in Martin et al.'s study (50), sarcopenia was defined as $<41 \mathrm{~cm}^{2} / \mathrm{m}^{2}$ in women, $<43 \mathrm{~cm}^{2} / \mathrm{m}^{2}$ in men with BMI $<25 \mathrm{~kg} / \mathrm{m}^{2}$, and $<$ $53 \mathrm{~cm}^{2} / \mathrm{m}^{2}$ in men with a BMI $>25 \mathrm{~kg} / \mathrm{m}^{2}$. In other studies using the psoas muscle and mid-thigh muscle, a diverse range of cutoffs have been used to diagnose sarcopenia (3). The diagnostic cutoff values must be standardized, but this requires further evidence from large-scale studies.

\section{Issues for CT/MRI Acquisition and Analysis}

In CT and MRI, there is no standardized protocol for image acquisition of body fat and muscle mass quantification. In particular, the measurement field (i.e., whole body scanning versus single-slice selection) and measurement area/level (i.e., which area or level should be used when using a single-slice selection for sarcopenia assessment) have not been standardized to date. Although the assessment of sarcopenia based on whole body imaging is the most accurate, it is very time consuming and costly; thus, it may not be practical in most clinical settings. Therefore, identifying which anatomical level or muscles best represents the total lean body mass is a very important issue. To date, there have been three main proposals: TAMA at the lumbar spine level; psoas muscle area in the lumbar spine level; and thigh muscles at the mid-thigh level, as illustrated in Figure 5 (35).

Currently, the most frequently used landmark among 
sectional body composition studies is the L3 level of the lumbar vertebra, used for the measurement of TAMA. At this level, the field of view includes the major large muscles and main functional muscles of the human body, which are the psoas, paraspinal muscles (erector spinae, quadratus lumborum) and abdominal wall muscles (transversus abdominus, external and internal obliques, and rectus abdominus), making it the optimal level for skeletal muscle analysis. In several studies, a single scan at the level of L3 was the best compromise site for assessing the total tissue volumes of skeletal muscle, visceral adipose tissue, and subcutaneous adipose tissue $(36,51-53)$. Schweitzer et al. $(51,54)$, investigated level L1 through L5 to determine the best estimates of skeletal muscle and visceral fat using single slice image of MRI, and confirmed that L3 level showed highest correlation with whole-body skeletal muscle and visceral fat volume.

However, many studies have also used the L 4 level and the mid-thigh level. A single-slice MR image at the mid femur level showed good estimation of skeletal muscle and fat volume in the thighs and correlations with clinical criteria for sarcopenia in older adults (55). Availability of multiple protocols for image acquisition limits the standardization of muscle mass and quality evaluation; thus, further large-scale studies and standardization of the measurement field/level are warranted.

In most recent studies that used CT/MRI for sarcopenia assessment, clinically acquired CT/MRI were used by applying additional imaging segmentation techniques to measure the muscle and fat mass. The imaging segmentation techniques vary according to the level of automation, such as a manual or semi-automatic, based on a threshold level, or an artificial intelligence-driven fully-automated technique (47). Recently, the AsanJ-Morphometry ${ }^{\mathrm{TM}}$ software (Asan Image Metrics, Seoul, Korea) was developed for abdominal muscle and fat area measurements based on ImageJ (NIH, Bethesda, MD, USA) and was made publicly available for non-profit research (available at http://datasharing.aim-

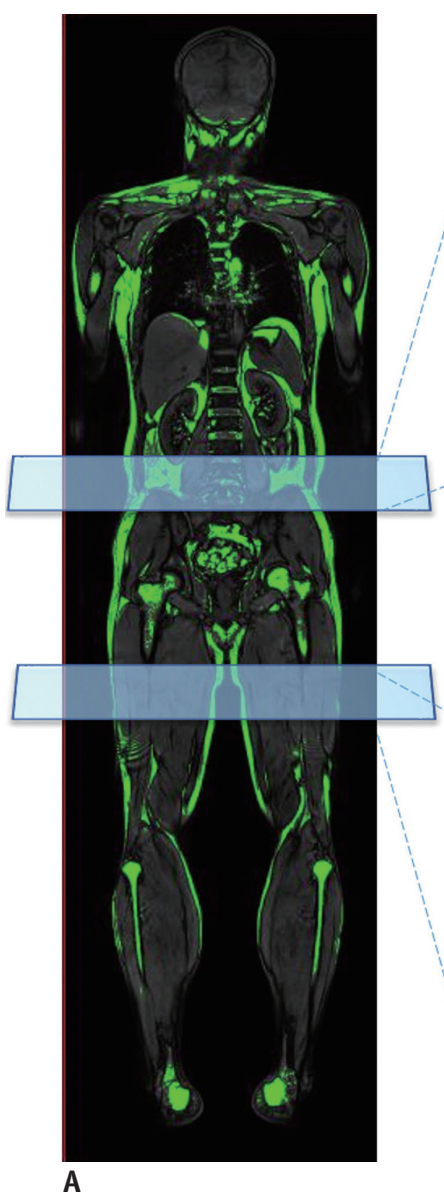

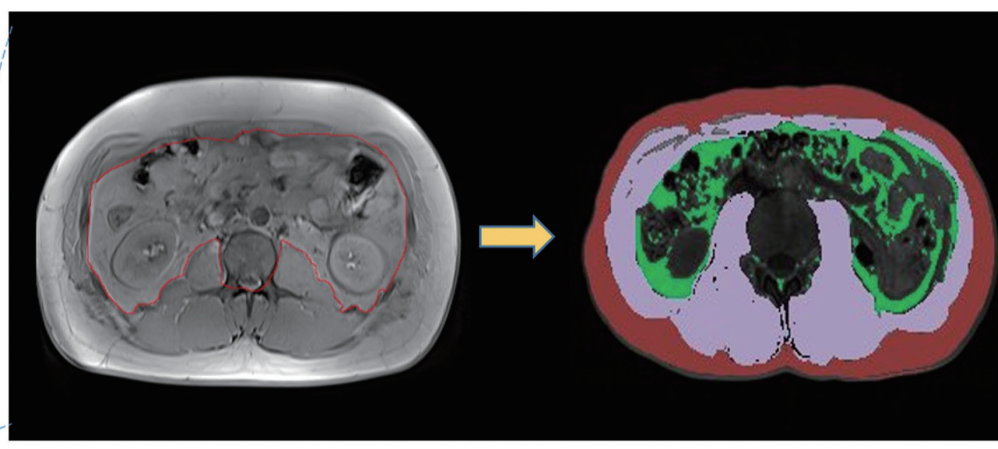

B

Skeletal muscle

Visceral fat

Subcutaneous fat

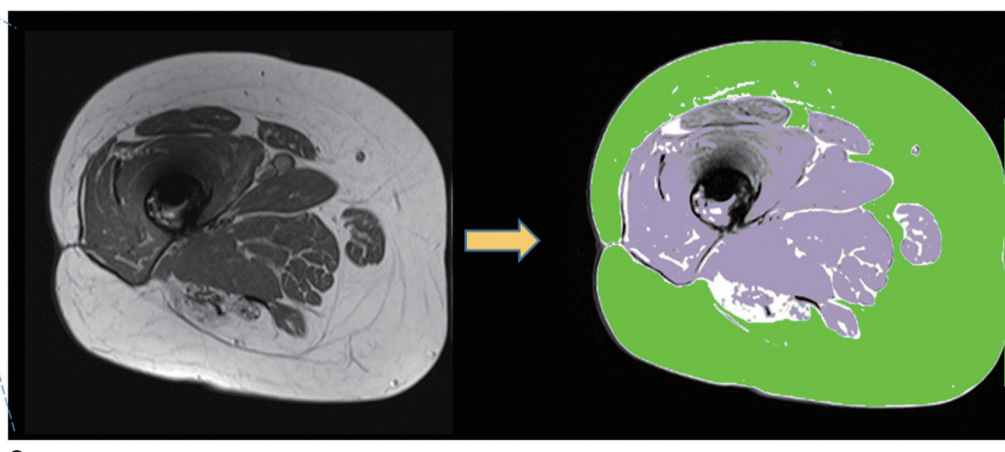

C

Fig. 5. Sarcopenia evaluation with MRI.

In clinically acquired whole-body MRI of 55-year-old male patient (A), TAMA is segmented at L3 vertebral body level (B) and thigh muscle area is segmented at mid-thigh level (C). 
aicro.com/morphometry).

\section{Quantitative Computed Tomography}

Recently, the use of new CT techniques, such as quantitative computed tomography (QCT), have been being investigated. Peripheral QCT (pQCT) is a novel imaging modality that has been primarily used to investigate bone mineral content. Like standard CT scans, PQCT produces a cross-sectional image that enables quantification of 3-dimensional tissue structure and skeletal muscle evaluation. But unlike general CT, pQCT uses a smaller scanner to study peripheral tissue. In the past, pQCT use was limited to the peripheral limb, usually the tibia and mid forearm (47). However, with technological advances, it became possible to analyze larger areas, up to the midthigh level. pQCT has the important advantages of extremely low radiation exposure, shorter scan time, and relatively low cost compared to whole body CT $(37,47)$.

In one study, Xu et al. (56) compared QCT and biochemical measurement of geese hepatic steatosis. QCT measurements of goose liver fat were shown to be accurate and reliable, and QCT was suggested to have potential in clinical settings (56).

One limitation of PQCT is the lack of a standardize analysis protocol. Since determination of the scanning region is performed by calculating the percentage of limb length from a reference line, comparisons between studies are difficult (47). Furthermore, pQCT scanning is limited to peripheral anatomical sites, which have lower accuracy compared to the sites used for MRI, making it difficult to distinguish individual muscles $(43,47)$. With these limitations, current PQCT use may be limited to situations where portability is necessary (55).

\section{Ultrasonography}

Although US has limitations for use in evaluating skeletal muscle, it can be a good option in some clinical settings for an initial evaluation of the quality and quantity of skeletal muscle mass (Fig. 6). Its major advantages as compared to other modalities are its low cost, portability, and lack of radiation exposure. In particular, its portability is particularly advantageous: unlike other modalities, whose lack of portability limit their use in large epidemiological studies, the portability of US yields a marked advantage in clinical settings, which explains its growing importance in studying skeletal muscle $(57,58)$. As no radiation is required, US can be used for all patients, including children and pregnant women. Another important advantage of US is that it allows real-time visualization of the target structure, and through echogenicity, it can provide information about the presence of inflammation, fibrosis, and adipose infiltration (57).

The main limitation of US is its lack of a standardized protocol and examiner-dependent factors, which can lead to evaluation errors and thus interfere with the reproducibility of results. Additionally, the numerous devices available commercially show great technological diversity (larger than that commonly found with DXA) and employ different methods for calibration, thus making it difficult to compare
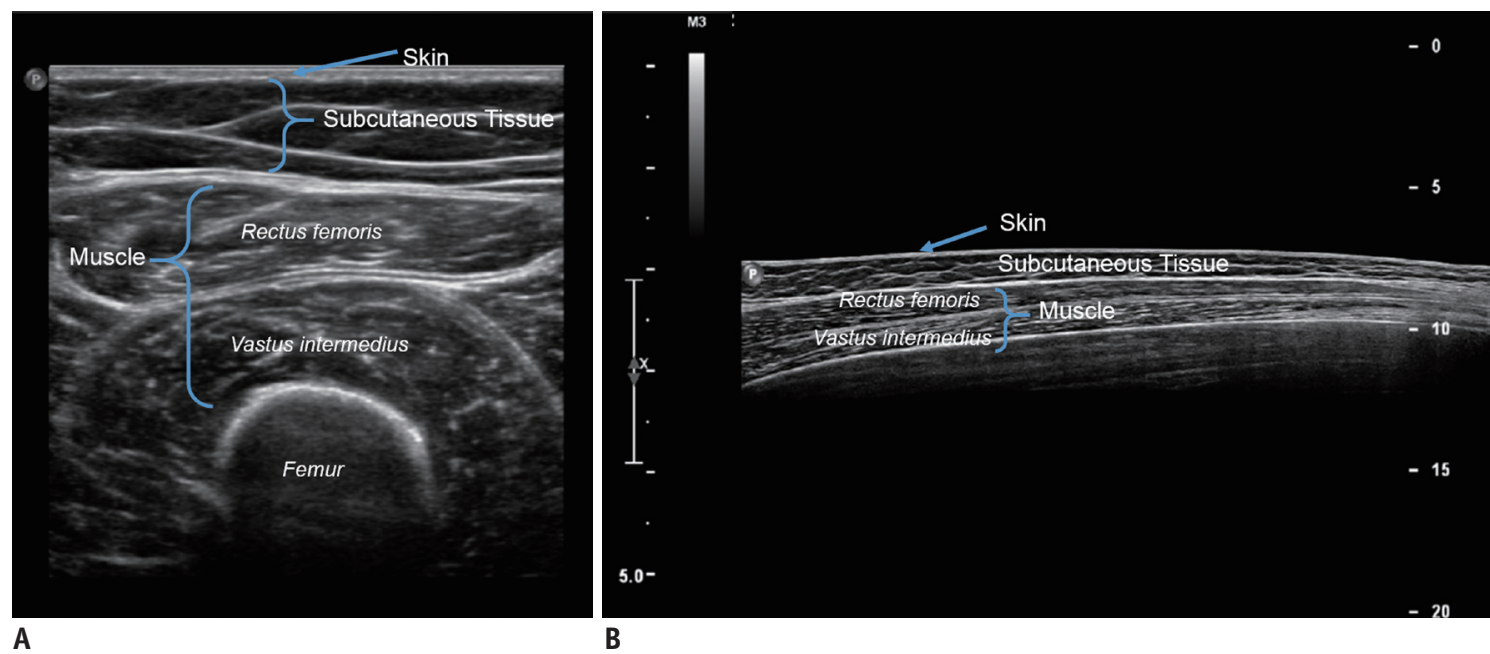

Fig. 6. US images of thigh muscle.

In clinically acquired axial view US image of 30-year-old female patient (A), and sagittal panoramic view (B) provides both qualitative and quantitative information of thigh muscle. US = ultrasonography 
results from different studies directly (37).

\section{CONCLUSION}

Body composition imaging has become increasingly common as the importance of sarcopenia has been recognized in clinical practice and research. Currently, DXA and CT/MRI are most commonly used imaging modalities for sarcopenia assessment. In general, DXA is utilized in the clinical setting of primary care for health surveillance/ screening, geriatric medicine, rehabilitation medicine, and endocrinology/metabolic disease. In contrast, CT/ MRI is used when CT/MRI is clinically acquired for disease diagnosis/treatment/follow-up, particularly in cancer patients or patients treated with major surgery. Because of their rapid and major technological advances, many tools are now available to diagnose and evaluate sarcopenia. However, there is a lack of consensus and standardization in the definition of sarcopenia, imaging modalities, measurement methods, and diagnostic cutoff values. Radiologists should be aware of the current updates on body composition imaging and the contentious issues, and should guide clinicians to utilize the imaging tool best suited for muscle mass assessment and patient management.

\section{Conflicts of Interest}

The authors have no financial conflicts of interest.

\section{ORCID}

Jimi Huh

https://orcid.org/0000-0002-8832-6165

Koeun Lee

https://orcid.org/0000-0001-8202-3641

Yongbin Shin

https://orcid.org/0000-0003-2753-9586

\section{REFERENCES}

1. Beaudart $C$, Zaaria M, Pasleau F, Reginster JY, Bruyère 0 . Health outcomes of sarcopenia: a systematic review and meta-analysis. PLoS One 2017;12:e0169548

2. Bazzocchi A, Diano D, Ponti F, Salizzoni E, Albisinni U, Marchesini G, et al. A 360-degree overview of body composition in healthy people: relationships among anthropometry, ultrasonography, and dual-energy x-ray absorptiometry. Nutrition 2014;30:696-701

3. Boutin RD, Yao L, Canter RJ, Lenchik L. Sarcopenia: current concepts and imaging implications. AJR Am J Roentgenol 2015;205:W255-W266

4. Santilli V, Bernetti A, Mangone M, Paoloni M. Clinical definition of sarcopenia. Clin Cases Miner Bone Metab 2014;11:177-180

5. Cruz-Jentoft AJ, Baeyens JP, Bauer JM, Boirie Y, Cederholm T, Landi F, et al. Sarcopenia: European consensus on definition and diagnosis: report of the European Working Group on sarcopenia in older people. Age Ageing 2010;39:412-423

6. Xue QL. The frailty syndrome: definition and natural history. Clin Geriatr Med 2011;27:1-15

7. Chen X, Mao G, Leng SX. Frailty syndrome: an overview. Clin Interv Aging 2014;9:433-441

8. Cederholm T. Overlaps between Frailty and sarcopenia definitions. Nestle Nutr Inst Workshop Ser 2015;83:65-69

9. Anandavadivelan P, Lagergren P. Cachexia in patients with oesophageal cancer. Nat Rev Clin Oncol 2016;13:185-198

10. Cruz-Jentoft AJ, Landi F, Schneider SM, Zúñiga C, Arai H, Boirie $Y$, et al. Prevalence of and interventions for sarcopenia in ageing adults: a systematic review. Report of the International Sarcopenia Initiative (EWGSOP and IWGS). Age Ageing 2014;43:748-759

11. Wang C, Bai L. Sarcopenia in the elderly: basic and clinical issues. Geriatr Gerontol Int 2012;12:388-396

12. Kaji H. Effects of myokines on bone. Bonekey Rep 2016;5:826

13. Laske C, Stransky E, Leyhe T, Eschweiler GW, Wittorf A, Richartz $E$, et al. Stage-dependent BDNF serum concentrations in Alzheimer's disease. J Neural Transm (Vienna) 2006;113:1217-1224

14. Pratesi A, Tarantini F, Di Bari M. Skeletal muscle: an endocrine organ. Clin Cases Miner Bone Metab 2013;10:11-14

15. Kim KM, Jang HC, Lim S. Differences among skeletal muscle mass indices derived from height-, weight-, and body mass index-adjusted models in assessing sarcopenia. Korean $\mathrm{J}$ Intern Med 2016;31:643-650

16. Chin S0, Rhee SY, Chon S, Hwang YC, Jeong IK, Oh S, et al. Sarcopenia is independently associated with cardiovascular disease in older Korean adults: the Korea National Health and Nutrition Examination Survey (KNHANES) from 2009. PLoS One 2013;8:e60119

17. Sanada K, Miyachi M, Tanimoto M, Yamamoto K, Murakami H, Okumura S, et al. A cross-sectional study of sarcopenia in Japanese men and women: reference values and association with cardiovascular risk factors. Eur J Appl Physiol 2010;110:57-65

18. Levolger S, van Vugt JL, de Bruin RW, IJzermans JN. Systematic review of sarcopenia in patients operated on for gastrointestinal and hepatopancreatobiliary malignancies. $\mathrm{Br}$ J Surg 2015;102:1448-1458

19. Oakland K, Nadler R, Cresswell L, Jackson D, Coughlin PA. Systematic review and meta-analysis of the association between frailty and outcome in surgical patients. Ann R Coll Surg Engl 2016;98:80-85

20. Englesbe MJ, Patel SP, He K, Lynch RJ, Schaubel DE, Harbaugh 
C, et al. Sarcopenia and mortality after liver transplantation. J Am Coll Surg 2010;211:271-278

21. Ganapathi AM, Englum BR, Hanna JM, Schechter MA, Gaca JG, Hurwitz LM, et al. Frailty and risk in proximal aortic surgery. $J$ Thorac Cardiovasc Surg 2014;147:186-191.e1

22. Lee JS, He K, Harbaugh CM, Schaubel DE, Sonnenday CJ, Wang SC, et al.; Michigan Analytic Morphomics Group (MAMG). Frailty, core muscle size, and mortality in patients undergoing open abdominal aortic aneurysm repair. J Vasc Surg 2011;53:912-917

23. Peng P, Hyder 0, Firoozmand A, Kneuertz P, Schulick RD, Huang $D$, et al. Impact of sarcopenia on outcomes following resection of pancreatic adenocarcinoma. J Gastrointest Surg 2012;16:1478-1486

24. de Hoogt PA, Reisinger KW, Tegels JJW, Bosmans JWAM, Tijssen F, Stoot JHMB. Functional Compromise Cohort Study (FCCS): sarcopenia is a strong predictor of mortality in the intensive care unit. World J Surg 2018;42:1733-1741

25. Du Y, Karvellas CJ, Baracos V, Williams DC, Khadaroo RG; Acute Care and Emergency Surgery (ACES) Group. Sarcopenia is a predictor of outcomes in very elderly patients undergoing emergency surgery. Surgery 2014;156:521-527

26. Miljkovic I, Zmuda JM. Epidemiology of myosteatosis. Curr Opin Clin Nutr Metab Care 2010;13:260-264

27. Reinders I, Murphy RA, Koster A, Brouwer IA, Visser M, Garcia ME, et al. Muscle quality and muscle fat infiltration in relation to incident mobility disability and gait speed decline: the age, gene/environment susceptibility-Reykjavik Study. J Gerontol A Biol Sci Med Sci 2015;70:1030-1036

28. Buford TW, Lott DJ, Marzetti E, Wohlgemuth SE, Vandenborne K, Pahor M, et al. Age-related differences in lower extremity tissue compartments and associations with physical function in older adults. Exp Gerontol 2012;47:38-44

29. Lorbergs AL, Noseworthy MD, Adachi JD, Stratford PW, MacIntyre NJ. Fat infiltration in the leg is associated with bone geometry and physical function in healthy older women. Calcif Tissue Int 2015;97:353-363

30. Hamrick MW, McGee-Lawrence ME, Frechette DM. Fatty infiltration of skeletal muscle: mechanisms and comparisons with bone marrow adiposity. Front Endocrinol (Lausanne) 2016;7:69

31. Montano-Loza AJ, Angulo P, Meza-Junco J, Prado CM, Sawyer MB, Beaumont C, et al. Sarcopenic obesity and myosteatosis are associated with higher mortality in patients with cirrhosis. J Cachexia Sarcopenia Muscle 2016;7:126-135

32. Rollins KE, Tewari N, Ackner A, Awwad A, Madhusudan $S$, Macdonald IA, et al. The impact of sarcopenia and myosteatosis on outcomes of unresectable pancreatic cancer or distal cholangiocarcinoma. Clin Nutr 2016;35:1103-1109

33. Reinders I, Murphy RA, Brouwer IA, Visser M, Launer L, Siggeirsdottir K, et al. Muscle quality and myosteatosis: novel associations with mortality risk: the age, gene/environment susceptibility (AGES)-Reykjavik Study. Am J Epidemiol 2016;183:53-60
34. Shepherd J. Evaluation of sarcopenia by DXA. Clin Rev Bone Miner Metab 2016;14:45-49

35. Morrell GR, Ikizler TA, Chen X, Heilbrun ME, Wei G, Boucher $R$, et al. Psoas muscle cross-sectional area as a measure of whole-body lean muscle mass in maintenance hemodialysis patients. J Ren Nutr 2016;26:258-264

36. Noumura Y, Kamishima T, Sutherland K, Nishimura H. Visceral adipose tissue area measurement at a single level: can it represent visceral adipose tissue volume? Br J Radiol 2017; $90: 20170253$

37. Guerri S, Mercatelli D, Aparisi Gómez MP, Napoli A, Battista G, Guglielmi G, et al. Quantitative imaging techniques for the assessment of osteoporosis and sarcopenia. Quant Imaging Med Surg 2018;8:60-85

38. Baumgartner RN, Koehler KM, Gallagher D, Romero L, Heymsfield SB, Ross RR, et al. Epidemiology of sarcopenia among the elderly in New Mexico. Am J Epidemiol 1998; 147:755-763

39. Fuggle N, Shaw S, Dennison E, Cooper C. Sarcopenia. Best Pract Res Clin Rheumatol 2017;31:218-242

40. Kim H, Hirano H, Edahiro A, Ohara Y, Watanabe Y, Kojima N, et al. Sarcopenia: prevalence and associated factors based on different suggested definitions in community-dwelling older adults. Geriatr Gerontol Int 2016;16 Suppl 1:110-122

41. Cawthon PM, Peters KW, Shardell MD, McLean RR, Dam TT, Kenny AM, et al. Cutpoints for low appendicular lean mass that identify older adults with clinically significant weakness. J Gerontol A Biol Sci Med Sci 2014;69:567-575

42. Seabolt LA, Welch EB, Silver HJ. Imaging methods for analyzing body composition in human obesity and cardiometabolic disease. Ann N Y Acad Sci 2015;1353:41-59

43. Sergi G, Trevisan C, Veronese N, Lucato P, Manzato E. Imaging of sarcopenia. Eur J Radiol 2016;85:1519-1524

44. Yoon DY, Moon JH, Kim HK, Choi CS, Chang SK, Yun EJ, et al. Comparison of low-dose CT and MR for measurement of intraabdominal adipose tissue: a phantom and human study. Acad Radiol 2008;15:62-70

45. Lustgarten MS, Fielding RA. Assessment of analytical methods used to measure changes in body composition in the elderly and recommendations for their use in phase II clinical trials. J Nutr Health Aging 2011;15:368-375

46. Dalakas MC. Inflammatory muscle diseases. N Engl J Med 2015;372:1734-1747

47. Erlandson MC, Lorbergs AL, Mathur S, Cheung AM. Muscle analysis using PQCT, DXA and MRI. Eur J Radiol 2016;85:15051511

48. Gloor M, Fasler S, Fischmann A, Haas T, Bieri 0, Heinimann $\mathrm{K}$, et al. Quantification of fat infiltration in oculopharyngeal muscular dystrophy: comparison of three MR imaging methods. J Magn Reson Imaging 2011;33:203-210

49. Prado CM, Lieffers JR, McCargar LJ, Reiman T, Sawyer MB, Martin L, et al. Prevalence and clinical implications of sarcopenic obesity in patients with solid tumours of the respiratory and gastrointestinal tracts: a population-based 
study. Lancet Oncol 2008;9:629-635

50. Martin L, Birdsell L, Macdonald N, Reiman T, Clandinin MT, McCargar LJ, et al. Cancer cachexia in the age of obesity: skeletal muscle depletion is a powerful prognostic factor, independent of body mass index. J Clin Oncol 2013;31:15391547

51. Schweitzer L, Geisler C, Pourhassan M, Braun W, Glüer CC, Bosy-Westphal A, et al. What is the best reference site for a single MRI slice to assess whole-body skeletal muscle and adipose tissue volumes in healthy adults? Am J Clin Nutr 2015;102:58-65

52. Yip C, Dinkel C, Mahajan A, Siddique M, Cook GJ, Goh V. Imaging body composition in cancer patients: visceral obesity, sarcopenia and sarcopenic obesity may impact on clinical outcome. Insights Imaging 2015;6:489-497

53. Cheng X, Zhang Y, Wang C, Deng W, Wang L, Duanmu Y, et al. The optimal anatomic site for a single slice to estimate the total volume of visceral adipose tissue by using the quantitative computed tomography (QCT) in Chinese population. Eur J Clin Nutr 2018 Mar 20 [Epub ahead of print]. https://doi.org/10.1038/s41430-018-0122-1
54. Schweitzer L, Geisler C, Pourhassan M, Braun W, Glüer CC, Bosy-Westphal A, et al. Estimation of skeletal muscle mass and visceral adipose tissue volume by a single magnetic resonance imaging slice in healthy elderly adults. J Nutr 2016;146:2143-2148

55. Tosato M, Marzetti E, Cesari M, Savera G, Miller RR, Bernabei $\mathrm{R}$, et al. Measurement of muscle mass in sarcopenia: from imaging to biochemical markers. Aging Clin Exp Res 2017;29:19-27

56. Xu L, Duanmu Y, Blake GM, Zhang C, Zhang Y, Brown K, et al. Validation of goose liver fat measurement by QCT and CSE-MRI with biochemical extraction and pathology as reference. Eur Radiol 2018;28:2003-2012

57. Ticinesi A, Meschi T, Narici MV, Lauretani F, Maggio M. Muscle ultrasound and sarcopenia in older individuals: a clinical perspective. J Am Med Dir Assoc 2017;18:290-300

58. Glüer CC. Quantitative ultrasound techniques for the assessment of osteoporosis: expert agreement on current status. The International Quantitative Ultrasound Consensus Group. J Bone Miner Res 1997;12:1280-1288 